\title{
BRCA1 mutations in a selected series of breast/ovarian cancer patients
}

Alex M Garvin, Martina Spycher, Michele Häner, Joachim Torhorst, Hansjakob Müller, Richard Herrmann, Christoph Rochlitz, Walter Weber, Rodney J Scott

\begin{abstract}
Germline mutations in the BRCA1 gene have been associated with familial breast/ ovarian cancer in large families showing high penetrance of the disease. Little is known, however, about the contribution of BRCA1 mutations to breast/ovarian cancer in small families with few affected members or in isolated early onset cases. Therefore we examined the BRCA1 gene in 63 breast/ovarian cancer patients who either came from small families with as few as one affected first degree relative, or in patients who had no family history but had developed breast cancer under 40 years of age. Using the protein truncation test, we were able to identify three unique BRCA1 germline mutations (4.8\%). Two of the probands had only one affected first degree and several second degree relatives and the third had three affected first degree relatives including two sisters who, when tested, were also found to carry the mutation. There was no family history of ovarian cancer in any of the three families.

(F Med Genet 1996;33:721-725)
\end{abstract}

Key words: breast cancer; BRCA1; mutations.

Research,

Kantonsspital Basel, Hebelstrasse 20, Basel CH-4031, Switzerland A M Garvin

M Spycher

$M$ Häner

H Müller

W Weber

R J Scott

Division of Oncology, Department of

Medicine,

Kantonsspital Basel,

Basel CH-4031,

Switzerland

R Herrmann

C Rochlitz

Institute for Pathology, Kantonsspital Basel, Basel CH-4031,

Switzerland

J Torhorst

Correspondence to: Dr Scott.

Received 27 February 1996 Revised version accepted for publication 2 May 1996 The BRCA1 gene was recently isolated ${ }^{6}$ and is believed to confer an $85 \%$ risk of developing breast cancer by the age of 70 years in women identified as harbouring germline mutations in this gene and a $63 \%$ risk of developing ovarian cancer by the same age. ${ }^{7}$ The BRCAl gene codes for a $7.5 \mathrm{~kb}$ transcript which is spread over $100 \mathrm{~kb}$ of genomic DNA. The coding sequence consists of 24 exons of which 22 are coding. The first 10 and the last 13 are relatively small, whereas exon 11 accounts for about $60 \%$ of the entire coding region of the gene. ${ }^{6}$

Mutation analysis of the BRCA1 gene shows a wide spectrum of mutations occurring throughout the gene with little evidence of mutational hot spots. There remains some debate as to whether a phenotypic gradient exists in the BRCA1 gene as mutations reported at the 3 ' end appear to predispose to breast cancer, whereas those towards the 5 ' end appear to be associated with an increased likelihood of ovarian cancer. ${ }^{8}$ Somatic BRCA1 mutations have not been found in breast tumours, ${ }^{9}$ but have been found in a small percentage of sporadic ovarian tumours. ${ }^{10}$ To date most BRCA1 mutations have been identified in large families where there is a high level of penetrance and consequently there is good evidence that a breast cancer susceptibility gene is involved. However, germline BRCA1 mutations should also account for some of the breast cancer cases found in people lacking an obvious family history. Small family size, paternal transmission, a high proportion of males, and incomplete or inaccurate family histories can obscure the presence of a breast cancer susceptibility gene in a given kindred.

We attempted to determine the role of BRCA1 in a group of 63 breast/ovarian cancer patients using selection criteria that maximised the probability of detecting germline mutations given that there was no strong family history. The entire BRCA1 coding region was examined using the protein truncation test and the mutations thus identified were sequenced. The results indicate that three of the 63 patients $(4.8 \%)$ had previously unidentified BRCA1 mutations which we believe are causative.

\section{Materials and methods}

PATIENT SELECTION

For this study we selected 63 women who had as minimum requirements (1) breast cancer 
Table 1 Disease characteristics of the 63 breast cancer families

Number of families with only breast cancer

24 families with only 1 breast cancer patient

21 families with only 2 breast cancer patients

6 families with only 3 breast cancer patients

3 families with only 4 breast car

7 bilateral breast cancer patients

Number of families with breast and ovarian cancer

1 family with 1 ovarian cancer patient ( 1 breast cancer)

2 families with 2 ovarian cancer patients ( 1 breast cancer)

63 total number of patients examined

under the age of 40 years (24 patients), (2) breast cancer under 50 with at least one first degree relative having breast cancer under 50 (37 families), (3) breast cancer under 50 with a first degree relative having ovarian cancer at any age (one family), and (4) ovarian cancer at any age with a first degree relative having ovarian cancer at any age (one family). Although not applied in these criteria, many of the index patients had affected second degree relatives. All patients signed a declaration of informed consent before entering this study. The family characteristics of the 63 index patients are presented in table 1 indicating the number of sporadic early onset and familial cases.

CELL LINES

EBV transformed B cell lines GM 13705, GM 13708, and GM 13713 were purchased from the Coriell Institute (Camden, New Jersey) and were grown in $\mathrm{RPMI} / 10 \%$ fetal calf serum.

TEMPLATE PREPARATION

Genomic DNA was prepared from lymphocytes obtained from $10 \mathrm{ml}$ EDTA blood using the protocol described by Miller et al. ${ }^{11}$ Total RNA was prepared from PBLs isolated by ficoll-hypac centrifugation (within 24 hours after donation) of $10 \mathrm{ml}$ heparin treated blood by the acid guanidinium isothiocyanatephenol-chloroform extraction method. ${ }^{12}$ cDNA was made using $10 \mu \mathrm{g}$ of total RNA, $500 \mathrm{ng}$ random hexamer, and 400 units of $M-M L V$ reverse transcriptase in a $50 \mu$ l volume according to the manufacturer's protocol (BRL Life Technologies).

PCR AND SEQUENCINC

Five overlapping fragments of the BRCA1 coding sequence were PCR amplified from either genomic DNA or cDNA, as shown in fig 1 , using the primers given in table 2 . Segments II, III, and IV were PCR amplified from $200 \mathrm{ng}$ of genomic DNA template in a $25 \mu \mathrm{l}$ reaction volume using standard conditions except that the primer concentration was $200 \mathrm{nmol} / \mathrm{l}$. The samples were cycled at $94^{\circ} \mathrm{C}$ for 30 seconds, $55^{\circ} \mathrm{C}$ for 30 seconds, $72^{\circ} \mathrm{C}$ for two minutes, 35 times. Segments I and V were amplified from cDNA using the following procedure: $0.5 \mu \mathrm{l}$ (5 - $10 \mathrm{ng}$ ) of cDNA was used in a nested PCR procedure in which the external primers (P1, P5 for segment I and P10, P13 for segment V) were included for 20 cycles of amplification in a $10 \mu \mathrm{l}$ reaction volume. A $1 \mu \mathrm{l}$ aliquot of this reaction was removed and used as template for an additional 30 cycles of amplification in a 25 $\mu l$ reaction in the presence of the internal primers. The temperature and time parameters were as above. Oligonucleotide primers were purchased from Microsynth (Balgach, Switzerland) and were based on the BRCA1 cDNA sequence (Genbank accession number U14680) except for P13 in the 3'UTR which was taken from Friedman et al. ${ }^{13}$ Dideoxy sequencing of the PCR products was performed using Thermosequenase (Amersham, Buckinghamshire, UK) and ${ }^{32} \mathrm{P}$ labelled primers according to the manufacturer's protocol.

\section{PROTEIN TRUNCATION TEST (PTT)}

Transcription and translation of segments $\mathrm{I}-\mathrm{V}$ was performed using $1 \mu \mathrm{l}$ of PCR product in a $5 \mu \mathrm{l}$ reaction using the TNT T7 coupled reticulocyte lysate system (Promega, Madison, WI). In each reaction $0.2 \mu \mathrm{l}$ of EasyTag ${ }^{35} \mathrm{~S}$ Methionine (NEN, Boston, Mass) were included for labelling purposes. Labelled protein products were size fractionated on $12 \%$ SDS-PAGE gels, fixed, dried, and exposed to Biomax film (Kodak, Rochester, New York).

\section{Results and discussion}

We screened the entire coding region of BRCA1 from 63 breast/ovarian cancer patients using either genomic DNA or cDNA as template. Recently, missense mutations in exons 11 and 13 of BRCA1 have been shown to result in the absence of mutant BRCA1 message, ${ }^{14}$ presumably because of nonsense mediated decay of RNA transcripts that leads to a reduction of mutant BRCA1 mRNA making it difficult to detect under normal conditions. We found, however, that when nested PCR is used, we could distinguish identical mutations in exon 11 using either $\mathrm{CDNA}$ or genomic DNA as template. To verify that we could identify mutations using cDNA as template we amplified segment IV of BRCA1 by nested PCR (using P14 and P15 as external primers) from two cell lines known to have truncating mutations (GM13705 and GM13713) and a negative control (GM13708). Since segment IV is also present as continuous sequence in genomic DNA within exon 11 , it was necessary to show that

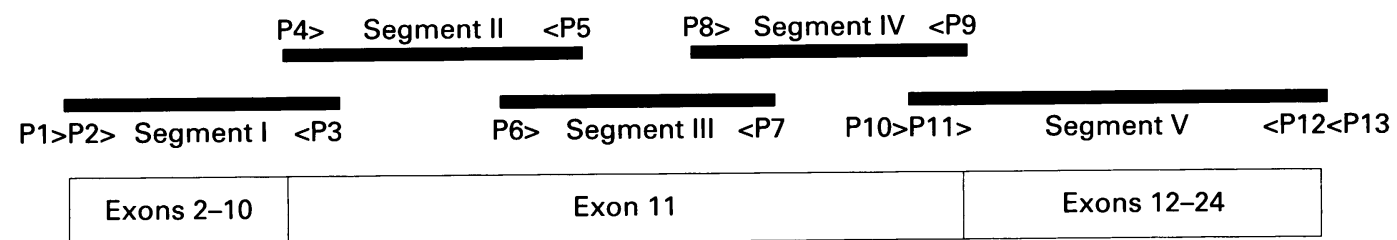

Figure 1 The coding sequence of BRCA1 is divided into five overlapping segments that can be amplified using the primer shown. 
Table 2 Primers used for PCR and sequencing

\begin{tabular}{lll}
\hline Primer & Sequence 5'-3' & Position in BRCA1 $^{\prime}$ \\
\hline P1 & TCTGCTCTGGGTAAAGTTCA & $85-104$ \\
P2 & T7-GATTTTCTGCTCTTCGCGT & $123-142$ \\
P3 & GTTCTCATGCTGTAATGAGC & $961-980^{\star}$ \\
P4 & T7-GCTTGTGAATTTTCTGAGAC & $792-811$ \\
P5 & TCATCACTTGACCATTCTGCTCC & $1736-1758^{\star}$ \\
P6 & T7-TTTGGGAAAACTATCGGAA & $1500-1519$ \\
P7 & CGTTGCCTCTGAACTGAGATGATAG & $2979-3003^{\star}$ \\
P8 & T7-CGCCAGTCATTTGCTCCGTTTTC & $2715-2737$ \\
P9 & TAAGTTTGATCCATGCTTTGC & $4193-4214^{\star}$ \\
P10 & TGTCTAAGAACACAGAGGAG & $3874-3893$ \\
P11 & T7-GCAAAGGCATCTCAGGAACATCA & $3948-3970$ \\
P12 & TCAGTAGTGGCTGTGGGGGATC & $5690-5711^{\star}$ \\
P13 & TAGCCAGGACAGTAGAAGGA & $3 ' \mathrm{UTR}$ \\
P14 & CCACAGTCGGGAAACAAGCA & $2633-2652$ \\
P15 & TGATGGAAGGGTAGCTGTTAGAAGG & $4392-4416^{\star}$ \\
P16 & CTTACTGGCCAGTGATCCTC & $1409-1428$ \\
P17 & ATTCAAGCAGAACTAGGTAG & $3321-3340$ \\
\hline
\end{tabular}

T7- refers to the T7 polymerase promoter and Kozak consensus

5'-GGATCCTAATACGACTCACTATAGGGAGACCACCATG-3'. The numbering of BRCA1 is that used in Genbank accession number U14680.

$\star=$ the reverse complement of the given BRCAl sequence.
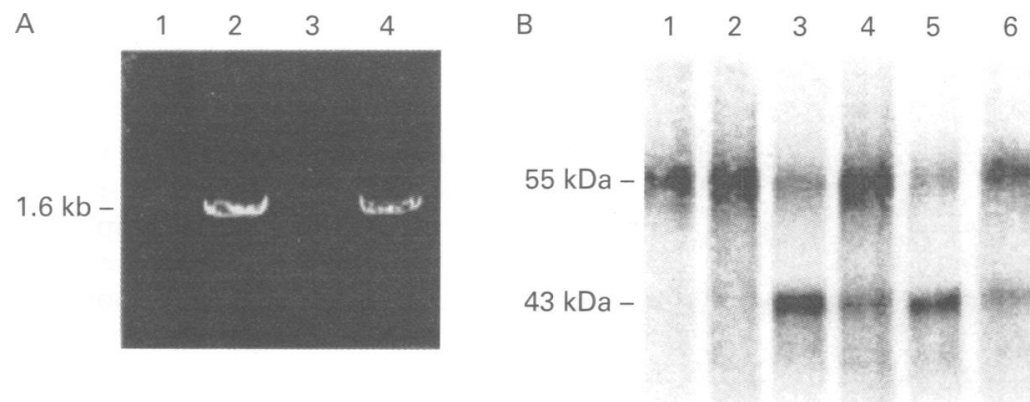

Figure 2 PTT of segment IV amplified from genomic DNA (lanes 1, 3, 5) or cDNA (lanes 2, 4, 6) derived from the negative control cell line GM13708 (lanes 1 and 2) and the mutant cell lines GM13705 (lanes 3 and 4) and GM13713 (lanes 5 and 6).

the template being amplified was cDNA and not contaminating genomic DNA. Wild type segment IV encodes a 500 residue polypeptide, whereas GM13705 and GM13713 cell lines are heterozygous for mutations that result in truncated products of 396 and 384 residues, respectively. The wild type and truncated proteins encoded by genomic DNA from these cell lines are seen in lanes 3 and 5 of fig 2. The same truncated proteins are also present in lanes 4 and 6 (although at slightly reduced levels), showing that BRCA1 mutations can be detected by PTT when cDNA is used as template.

Genomic DNA and cDNA from our series of patients were used to amplify segments I-V of BRCA1, and the PCR products were analysed by PTT. PTT should detect those BRCA1 mutations (approximately $90 \%$ of the total) which result in premature translational termination. In our series we detected three terminated proteins. Two patients had truncated products generated by segment II (lanes
A

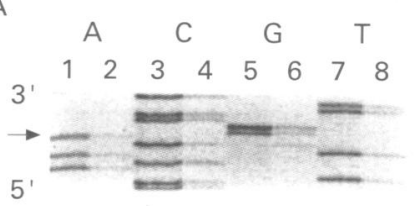

$B$

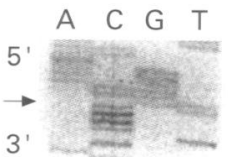

C

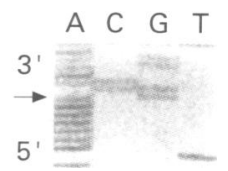

Figure 4 Sequences of the three BRCA1 mutations. (A) Segment II of patient 10 (even lanes) and a control (odd lanes) sequenced with oligonucleotide P16. Position C 1648 is indicated by the arrow. (B) Segment II of patient 46 sequenced with P5. C 1611 is indicated. (C) Segment IV of patient 20 sequenced with P17. The additional $A$ residue at position 3449 is indicated by an arrow.

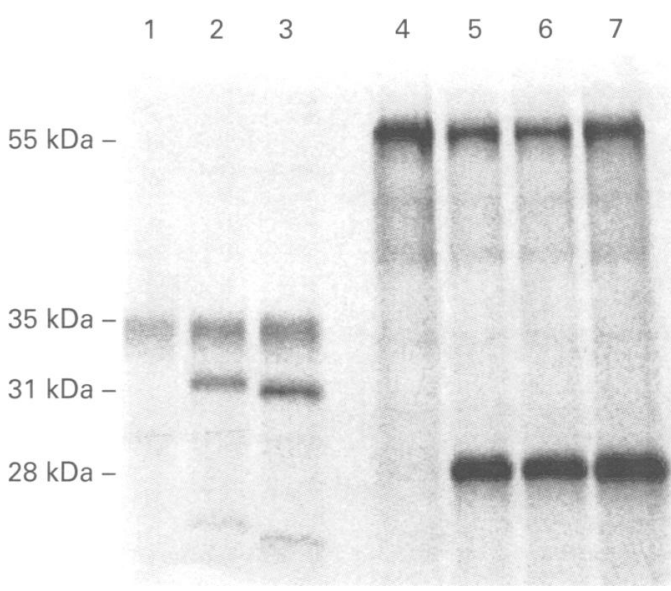

Figure 3 Three unique mutations in BRCA1 detected by PTT. Segment II (lanes 1, 2, 3) from a control (lane 1) and two patients (lanes 2 and 3) and segment IV (lanes 4-7) from a control (lane 4), patient 20 (lane 5), and two of her sisters (lanes 6 and 7).

2 and 3 of fig 3), and one patient had a truncated segment IV product (lane 5). This patient has two sisters with breast cancer who, when tested, were found to harbour the same mutation (lanes 6 and 7).

The sizes of the truncated products were used to estimate the positions of the mutations within each segment, and the heterozygous PCR products containing the mutations were sequenced. Patient 10 has a $\mathrm{C} \rightarrow \mathrm{G}$ transversion at base 1648 (fig 4A). The transversion results in a Ser 510 (TCA) Stop 510 (TGA) nonsense mutation which generates a segment II protein of 288 residues, 34 amino acids shorter than the wild type product. Patient 46 harbours a frameshift deletion of $C$, position 1611 (fig 4B). The frameshift introduces a stop codon 12 bases 3 ' to the deletion, resulting in a truncated segment II protein of 279 residues. Patient 19 has an additional A residue inserted in the stretch of six As between bases 3443 and 3448 (fig $4 \mathrm{C}$ ). The reading frameshift introduces a stop codon 18 bases 3 to the insertion and results in a segment IV product truncated by 247 residues to a size of 253 residues.

The criteria used for choosing patients include those having one or more first degree relatives with breast/ovarian cancer. The pedigrees of the three families (fig 5) show that each of the BRCA1 carriers does have affected first degree relatives. In each case the mutation appears to be maternally transmitted, perhaps reflecting a bias introduced in the selection criteria (paternally transmitted mutations would not result in an affected parent). Furthermore, our results confirm the finding that only persons with a family history of breast/ovarian cancer appear to have mutations.

Our BRCA1 mutation detection frequency of $4.8 \%$ (three of 63 ) is 38 fold higher than the $0.125 \%$ frequency of BRCA1 carriers in the general population calculated by Ford et $a l^{15}$ using population based studies of cancer mortality in the relatives of breast and ovarian cancer patients. Ford et $a l^{15}$ also estimated that BRCA1 mutations are causative in $5.3 \%$ of all breast cancers diagnosed before the age of 40 , yet only $1.7 \%$ of all breast cancers diagnosed before the age of $70 .{ }^{15}$ Recently, Fitzgerald et 
A

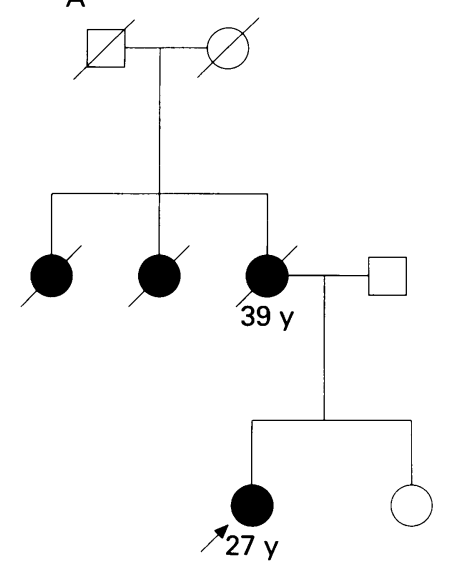

B

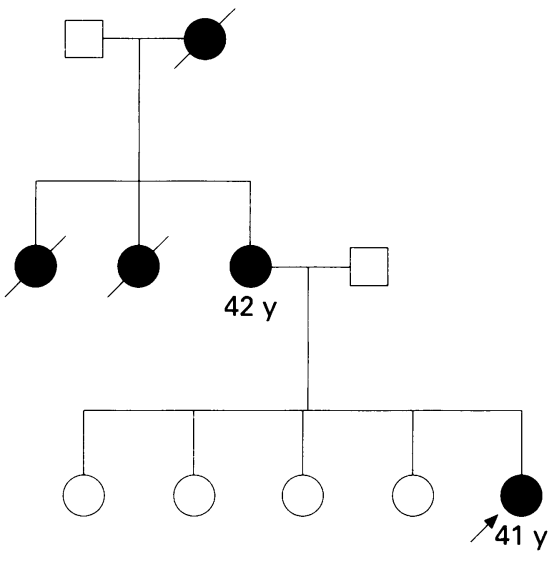

C

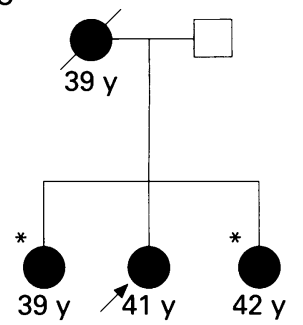

Figure 5 Pedigrees of $(A)$ patient 10, $(B)$ patient 16, $(C)$ patient 20 . The index patients are indicated by arrows and the age of diagnosis (dark symbols) is given when known. The two affected sisters of patient 20 are indicated by asterisks.

$a l^{16}$ performed a population based BRCA1 screening of women with breast cancer under the age of 30 and found that five of $30(17 \%)$ women harboured BRCA1 mutations, while Langston $e t$ al ${ }^{17}$ detected BRCA1 mutations in $7.5 \%$ of 80 women having breast cancer under the age of 35 . We tested patients ranging in age from 27 to 74 years with an average age of 41.5 years and detected BRCA1 mutations at a frequency similar to that expected in all women under 40 years of age with breast cancer, regardless of family history. The selection criteria in this study were aimed at small families and sporadic early onset breast cancer patients, whereas previous studies relied on large well documented families that had high a priori probabilities of being linked to BRCA1. The results of this investigation indicate that it is worthwhile studying a selected series of women based on restrictive criteria, as the incidence of BRCA1 mutations appears to be similar to that found in other investigations based on large informative families.

Given that our frequency of mutation detection is in accordance with that estimated, it appears that the PTT is a valuable method for rapidly screening the BRCA1 gene. Nevertheless, it is important to note that we did not compare this method of detection to any other and thus do not know if mutations were missed. In a recent study by Dobbie et $a l,{ }^{18}$ using the PTT to detect mutations in the adenomatous polyposis coli (APC) gene, it was found that this method could only identify $66 \%$ of all chain terminating mutations even though genomic DNA was used as template. Notwithstanding, given the size and structure of the BRCA1 gene we conclude that PTT screening is a rapid and efficient way of detecting BRCA1 mutations in subjects lacking or having an unconvincing family history of breast/ovarian cancer.

Although the breast/ovarian cancer patients used in this study fulfilled our selection criteria, only a small percentage of them appeared to harbour mutations in BRCA1. Those persons who did carry BRCA1 mutations came from families where there is a clear clustering of breast cancer at young ages in both first and second degree relatives. In the remaining families where the majority of patients were isolated cases under 40 years of age, it appears, from this limited study, that BRCA1 does not account for early onset disease in the absence of a substantial family history. Sporadic early onset breast cancer may well be the result of other factors affecting proliferating breast tissue, such as exposure to ionising radiation. ${ }^{19}$

In conclusion, given that only relatively few persons in this study are carriers of BRCA1 mutations and that virtually all BRCA1 mutations described to date appear to be associated with a familial clustering of breast/ovarian cancer, we would recommend that only women with a positive family history of early disease be considered for BRCA1 screening. Sporadic early onset breast cancer patients (those under 40 years of age) represent a special problem in relation to BRCA1 screening (especially in light of the paucity of de novo BRCA1 mutations). From this limited study it appears that BRCA1 mutations do not play a major role in early breast cancer susceptibility in women without a family history. Testing for other breast cancer susceptibility genes, such as BRCA2, and the ataxia telangiectasia gene (ATM) in this instance may be worthwhile.

This work was supported by grants AKT78, AKT332 and AKT463 from the Swiss Cancer League and by No 3200 042558.94 from the Swiss National Fund.

1 Newman B, Austin MA, Lee M, King MC. Inheritance of human breast cancer: evidence for autosomal dominance transmission in high-risk families. Proc Natl Acad Sci USA 1988;85:3044-8.

2 Black DM. The genetics of breast cancer. Eur 7 Cancer 1994;13:1957-61.

3 Narod SA, Amos C. Estimating the power of linkage analysis in hereditary breast cancer. Am $\mathcal{f}$ Hum Genet 1990;46:266-72.

4 Hall JM, Lee MK, Newman B, et al. Linkage of early-onset familial breast cancer to chromosome 17q21. Science 1990;250:1684-9.

5 Easton DF, Bishop DT, Ford D, Crockford GP. Genetic linkage analysis in familial breast and ovarian cancer: linkage analysis in familial breast and ovarian cancer:
results from 214 families. The Breast Cancer Linkage results from 214 families. The Breast Cancer
Consortium. Am f Hum Genet 1993;52:678-701.

6 Miki Y, Swensen J, Shattuck-Eidens D, et al. A strong candidate for the breast and ovarian cancer susceptibility gene BRCA1. Science 1994;266:66-71.

7 Easton DF, Ford D, Bishop DT, Consortium BC. Breast and ovarian cancer incidence in BRCA1-mutation carriers. Am ₹ Hum Genet 1995;56:265-71.

8 Gayther SA, Warren W, Mazoyer S, et al. Germline mutations of the BRCAl gene in breast and ovarian families provide evidence for a genotype-phenotype correlation. Nature Genet 1995;11:428-33.

9 Futreal PA, Liu Q, Shattuck-Eidens D, et al. BRCA1 mutations in primary breast and ovarian carcinomas. Science 1994;266:120-2.

10 Merajver SD, Pham TM, Caduff RF, et al. Somatic mutations in the BRCAl gene in sporadic ovarian mutations in the BRCA1 gene in

11 Miller SA, Dykes DD, Polesky HF. A simple salting out procedure for extracting DNA from human nucleated cells. cedure for extracting DNA from
Nucleic Acids Res 1988;53:739.

12 Chomczynski P, Sacchi N. Single step method of RNA isolation by acid guanidinium thiocyanate-phenolchloroform extraction. Anal Biochem 1987;162:156-9.

13 Friedman LS, Ostermeyer EA, Szabo CI, et al. Confirmation of BRCA1 by analysis of germline mutations linked to breast and ovarian cancer in ten families. Nature Genet 1994;8:399-404. 
14 Friedman, LS, Szabo CI, Ostermeyer EA, et al. Novel inherited mutations and variable expressivity of BRCA alleles, including the founder mutations 185delAG in Ashkenazi Jewish families. Am f Hum Genet 1995; 57:1284-97.

15 Ford D, Easton DF, Peto J. Estimates of the gene frequency of BRCA1 and its contribution to breast and ovarian cancer incidence. Am f Hum Genet 1995;57:1457-62.

16 Fitzgerald MG, MacDonald DJ, Krainer M, et al. Germ-line mutations in Jewish and non-Jewish women with earlymutations in Jewish and non-Jewish women with
onset breast cancer. $N$ Engl $\mathcal{H}$ Med 1996;334:143-9.
17 Langston AA, Malone KE, Thompson JD, Daling JR, Ostrander EA. BRCA1 mutations in a population-based sample of young women with breast cancer. $N$ Engl $\mathcal{F} M e d$ 1996;334:137-42.

18 Dobbie Z, Spycher M, Mary JL, et al. Correlation between the development of extracolonic manifestations in FAP patients and mutations beyond codon 1403 in the APC gene. F Med Genet 1996;33:274-80.

19 Bhatia S, Rodison LL, Oberlin O, et al. Breast cancer and other second neoplasms after childhood Hodgkin's disease. N Engl f Med 1996;334:747-51. 